No. $60313:: \quad$ The final report

\title{
Radiation Effects on Transport and Bubble Formation in Silicate Glasses
}

\author{
A. D. Trifunac, I. A. Shkrob, and D. W. Werst. \\ Chemistry Division, Argonne National Laboratory, 9700 S. Cass Avenue, Argonne, IL \\ 60439
}

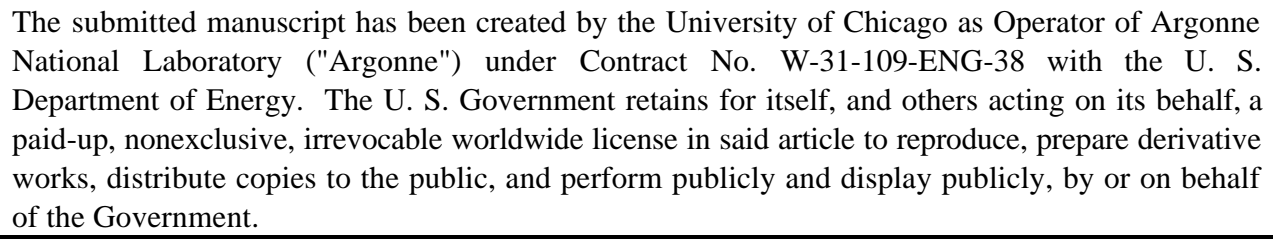

\section{Synopsis}

Using advanced magnetic resonance spectroscopies and small-cluster modeling, atomic structure of radiation-induced point defects in alkali borate, silicate, and borosilicate glasses is fully characterized. It is shown that in boron-containing glasses, most of these point defects are electrons/holes trapped by cation/anion vacancies, such as $\mathrm{O}_{1}{ }^{-}-\mathrm{O}_{3}{ }^{+}$valence-alternation pairs. In microscopically phase-separated borosilicate glasses, radiation-induced defects are found to cluster at the interface between the borate and silicate phases. Reaction and diffusion dynamics of defect-annealing interstitial hydrogen atoms in boron and silica oxide glasses are studied. The yield of radiolytic $\mathrm{O}_{2}$ is estimated. This oxygen is shown to be the final product of triplet exciton decay. Plausible mechanisms for the oxygen bubble formation are put forward. Two practical conclusions relevant for the EMSP mission are made: First, the yield of radiolytic oxygen is shown to be too low to interfere with the storage of vitrified radioactive waste in the first 10 Kyr. Second, microscopic phase separation is demonstrated to increase both the chemical and radiation stability of borosilicate glass.

\section{Funding and Support.}

This work was performed with financial support from the EMSP office of the US-DOE, grant No 60313 (a three-year program that started in 1997). The cost of equipment and facilities was supported by the BES office, Division of Chemical Science, US-DOE, under contract number W-31-109-ENG-38.

\section{Availability.}

The results are published in the following periodicals: 
I. A. Shkrob, B. M. Tadjikov, S. Chemerisov, and A. D. Trifunac, Electron Trapping and Hydrogen Atoms in Oxide Glasses, J. Chem. Phys. 111 (1999) 5124.

I. A. Shkrob, B. M. Tadjikov, and A. D. Trifunac, Magnetic Resonance Studies on Radiation-Induced Point Defects in Mixed Oxide Glasses. I. Spin Centers in $\mathrm{B}_{2} \mathrm{O}_{3}$ and Alkali Borate Glasses, J. Non-Cryst. Solids 262 (2000) 6-34.

I. A. Shkrob, B. M. Tadjikov, and A. D. Trifunac, Magnetic Resonance Studies on Radiation-Induced Point Defects in Mixed Oxide Glasses. II. Spin Centers in Alkali Silicate Glasses, J. Non-Cryst. Solids 262 (2000) 35-65.

I. A. Shkrob and V. F. Tarasov, On the Structure of Trapped Holes in Borosilicates, J. Chem. Phys. 113 (2000) 10723.

Two more publications are currently in preparation.

\section{Introduction}

Current scenarios for the long-term disposal of High-Level Radioactive Waste (HLW) involve immobilization of radionuclides in glass forms and placement of glass logs in a deep geologic repository. HLW forms must be highly durable and leach resistant to prevent release of radionuclide ions into the environment. The leach resistance must be maintained in spite of radiation damage caused by radionuclide decay. The HLW forms are alkali borosilicate glasses containing 20-50 wt $\%$ of transition metal oxides and 10-20 wt $\%$ of radionuclides. The most damaging radiation will include $\beta$ and $\gamma$ emission from ${ }^{137} \mathrm{Cs}$ and ${ }^{90} \mathrm{Sr}$ and other fission products and $\alpha$ emission from ${ }^{241} \mathrm{Am}$ and isotopes of $\mathrm{Pu}$. While $\beta$ and $\gamma$ radiation yields defects through ionization of the glass network, $\alpha$ particles displace atoms in energetic collisions. During the first $\mathrm{Kyr}$, when the radiation is most intense, it is dominated by $\beta$ and $\gamma$ emissions. At later times, the $\alpha$ emission takes over.

In the first 1-10 Kyr of storage, the HLW glasses will accumulate 0.1-10 Tera-rad (109$10^{11} \mathrm{~J} / \mathrm{kg}$ ) of ionizing radiation, not counting knock-on collisions with $\alpha$ particles and fission fragments. Eventual contact of glass chips with water will occur after 10-100 Kyr of storage; leaching of radionuclide ions by seeping water will then become a problem. One needs both a short-term (1-10 Kyr) and a long-term (0.1-1 Myr) prognosis of the glass durability and leaching resistance.

The concern is that the rates of glass aging/dissolution may change dramatically after 1-2 Kyr of storage, when every atom in the glass matrix has been ionized and displaced $>10^{6}$ times over. Though the dose rates in the HLW forms are not exceptional, over the geological time these doses sum up to the levels that are $10^{2}-10^{4}$ times higher than the radiation doses commonly studied by chemists, materials scientists, and geophysicists. It is unknown how amorphous solids in general and oxide glasses in particular will behave after absorbing these very high doses. 
The consensus of the US DOE panel scientists is that "at this stage a considerable fraction of the research effort should be focused on simple systems, such as alkali borates, silicates and borosilicates. At later stages, with more insight in the molecular mechanisms of radiolytic processes, more complex composite glasses can be studied." (Weber et al., J. Mater. Res. 12, 1946 (1997); US DOE, Council on Materials Science, Santa Fe, NM, February 2529, 1996).

A serious concern about Tera-rad glasses is the formation of microscopic gas bubbles and devitrification. In many oxide materials, radiolytic $\mathrm{O}_{2}$ coalesces to form $0.01-0.1 \mu \mathrm{m}$ bubbles. In the HLW forms produced at the Savannah River Site DWPF facility this process is vigorous, with several per cent of network oxygens converting to oxygen at 0.1-1 Tera-rad. The mechanism of this process is not known; there is also a controversy about the dose rate of bubble formation for different types of ionizing radiation. Finding the mechanism for production and agglomeration of interstitial oxygen is one of the highest priorities in the research on the HLW forms.

\section{Objectives.}

Our aim is to develop fundamental knowledge of radiation-matter interaction at the atomic level and provide mechanistic insight required for prognostication of the Tera-rad damage in HLW glasses through

- $\quad$ understanding of the mechanism for radiolytic damage at the atomic scale;

- $\quad$ quantifying the effects of radiation on generation, secondary reactions, migration and agglomeration of defects;

- $\quad$ linking of specific defects with the undesirable endpoints;

- $\quad$ studying the effects of glass composition and microstructure on the nature of radiolytic damage.

The following directions have been pursued:

- $\quad$ Studies of short-lived reactive precursors of point defects, such as electrons, holes, and mobile interstitial atoms;

- Identification and structural characterization of metastable point defects;

- Solving questions concerning oxygen bubble formation:

- resolving the controversy about the rates of oxygen formation;

- identification of the mechanism for oxygen formation and coalescence;

- identification of reduced phases in glasses.

\section{Oxygen bubbles: the yield.}


Radiation-induced evolution of $\mathrm{O}_{2}$ was first observed in the TV tubes. After 2 weeks of exposure to a typical $20 \mathrm{keV}$ electron beam (the dose of 4 Tera-rad), 10\% of network oxygens in a 2-3 $\mu \mathrm{m}$ layer near the surface was converted to $\mathrm{O}_{2}$. The yield of $\mathrm{O}_{2}$ was (1-3) $\times 10^{-6}$ molecules per electron volt of absorbed radiation. From TEM, the average radius of bubbles is $10-50 \mathrm{~nm}$, and their concentration at saturation is $(1-5) \times 10^{18} \mathrm{~cm}^{-3}$. The pressure inside the bubble is nearly-critical ( $>10^{4}$ molecules per bubble). Such agglomeration is possible if only the solid is saturated many times over by $\mathrm{O}_{2}$. For that, the yield of $\mathrm{O}_{2}$ must be $>10$ times greater than the concentration of $\mathrm{O}_{2}$ at saturation, $10^{17} \mathrm{~cm}^{-3}$, which corresponds to the dose of 0.01 0.1 Tera-rad. At lower doses, radiolytic $\mathrm{O}_{2}$ is present as interstitial gas, and no bubbles are formed.

Recently, a claim was made that the threshold for the bubble formation is lowered 4-5 orders of magnitude when the HLW glass is irradiated with $1.5 \mathrm{MeV} \gamma$-rays instead of 1-500 $\mathrm{keV}$ electrons. From these data, the dose rate of $\mathrm{O}_{2}$ formation should exceed 0.1 molecules per $\mathrm{eV}$. Some authors used this report to express concerns about short-term stability of the HLW forms. Others questioned the validity of this TEM study. During a routine TEM scan, the $\beta$ dose absorbed by a specimen exceeds the $\gamma$ dose by several orders of the magnitude.

In our study, glasses of various compositions were irradiated with $3 \mathrm{MeV}$ electrons to $(1-10) \times 10^{9} \mathrm{rad}$. For this energy, there should be little difference in radiation chemistry induced by $\beta$ - and $\gamma$-particles. To measure the gas evolution, $10-50 \mu \mathrm{m}$ glass powder was baked out in vacuo at $600^{\circ} \mathrm{C}$ and irradiated at $100-200^{\circ} \mathrm{C}$ using the electron beam from a $3 \mathrm{MeV}$ Van de Graaff accelerator. The evolved gases were analyzed using MS. The gas composition was studied as a function of the sample temperature, etc.

It was found that the yield of radiolytic $\mathrm{O}_{2}$ is lower than the lowest of the earlier estimates. Apparently, accumulation of negatively charged defects ("the space charge") that are formed when low-energy (1-to-100 keV) electrons are stopped inside the glass speeds up volatilization of the glass enormously. The $\mathrm{MeV}$ electrons have far greater penetration depths (centimeters) than the $\mathrm{keV}$ electrons (micrometers). Thus, homogeneous irradiation of glass with $\mathrm{MeV}$ electrons reduces the space charge by many orders of magnitude. Our results agree with the recent Raman spectroscopy studies (Boizot et al., J. Non-Cryst. Solids 243, 268 (1999)) in which stretching vibrations of interstitial $\mathrm{O}_{2}$ at $1550 \mathrm{~cm}^{-1}$ where observed; from these Raman measurements and our data on outgassing, the yield of $\mathrm{O}_{2}$ is $<10^{-7}$ molecules per eV. Since self-irradiation of the HLW glasses is homogeneous and the ionizing particles emitted have energies $>1 \mathrm{MeV}$, the radiolytic yield of $\mathrm{O}_{2}$ in the waste forms is quite low. Additional decrease is possible due to uptake of oxygen by transition metal ions present in the glass form.

In the dose regime studied, most of the evolved gases are formed due to radiolytic desorption of atmospheric gases and activation of chemisorbed $\mathrm{H}_{2} \mathrm{O}$ and $\mathrm{CO}_{2}$. The yield of radiolytic $\mathrm{O}_{2}$ is comparable to the yield of radiolytically desorbed $\mathrm{N}_{2}$ and by $10-100$ times less than the yield of $\mathrm{CO}_{2}$. Particularly low yields of $\mathrm{O}_{2}$ were observed in the simulated HLW glass 
MCC-76-68 from PNNL, due to uptake of $\mathrm{O}_{2}$ by transition metal ions. We found no evidence of efficient formation of $\mathrm{O}_{2}$ by high-energy radiation.

The implication of our study is that the concentration of $\mathrm{O}_{2}$ at which the bubbles are formed will accumulate only after $>10 \mathrm{Kyr}$ of the storage. There is no need to be concerned about volatilization of the HLW glasses in the nearest future.

\section{Point Defects: Structural aspects.}

Formation of atomic-scale ("point") defects is the first step towards the radiation damage of glass network. It is also the stage at which radiation chemistry plays the decisive role. In the past, a number of radiation-induced defects had been identified; however, little was known about their nature, structure, and origin. Using advanced magnetic resonance techniques, we systematically studied radiation-induced defects in alkali and alkaline earth borates, silicates, and borosilicates; related minerals have also been studied. Our work yields detailed information about the atomic structure and environment of these paramagnetic centers. In the following, we summarize some of these results; more detailed accounts could be found in the recent publications from our group (section 3).

\subsection{Alkali silicates and microscopic mechanisms for oxygen formation.}

Irradiation of alkali silicate glasses yields three classes of paramagnetic defects: oxygen hole centers, silicon dangling bonds, and peroxy radicals. The latter two defects are formed via dissociation of triplet excitons; the electron centers are negative- $U$ diamagnetic alkali metal clusters.

Previously, it has been repeatedly speculated that the radiation damage of silicate glass network is triggered by release of mobile alkali cations from holes trapped by $\equiv \mathrm{SiO}^{-} \mathrm{Alk}^{+}$ groups. We have shown that the hole trapping does not actually result in the cation release:

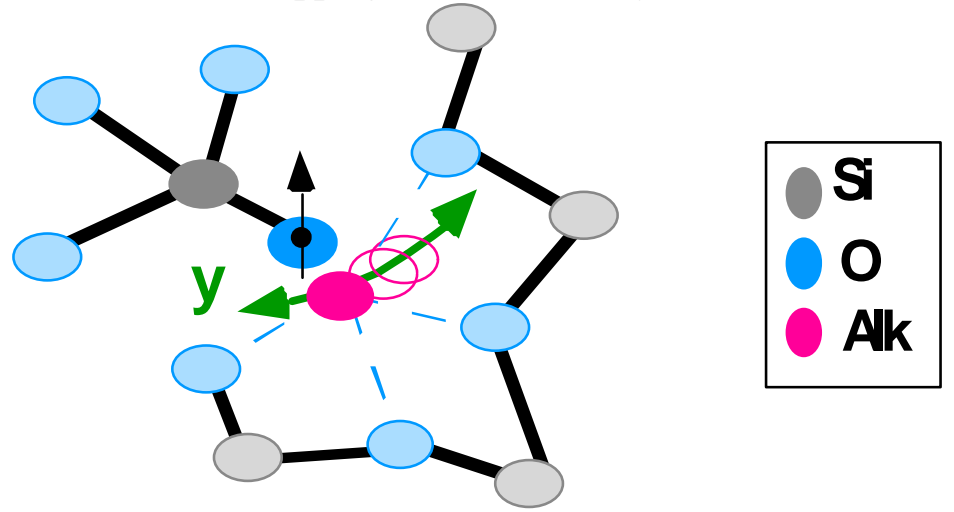

In the $\equiv \mathrm{SiO} \cdot \mathrm{Alk}^{+}$(oxygen hole) center, the O-Alk distance is just $10 \%$ longer than this distance in a regular $\equiv \mathrm{SiO}^{-} \mathrm{Alk}^{+}$group. Inside the cage formed by bridging oxygens, the cation undergoes thermally activated small-amplitude swinging motion along the $y$ axis. 

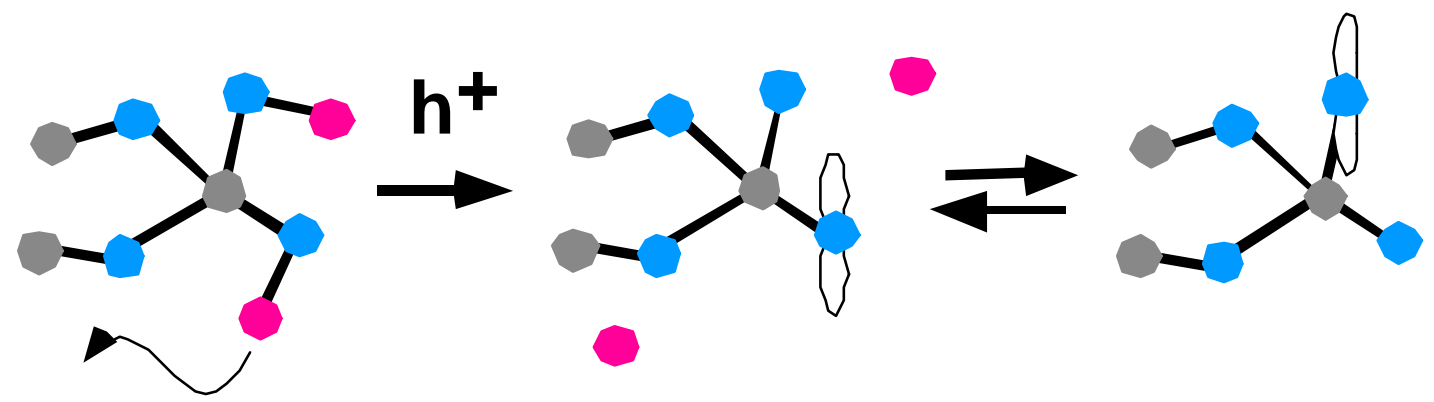

In alkali-loaded silicates, $=\mathrm{SiO}_{2}{ }^{2-}$ groups also trap holes; in these Jahn-Teller active centers the unpaired electron rapidly tunnels between two non-bridging oxygens, as shown above. The observation of such tunneling in our experiments solves a long-term controversy as to the nature of these hole centers.

Irradiation of alkali silicates yields trapped excitons; silicon peroxy radicals and silicon dangling bond centers are formed when these trapped triplet excitons dissociate:

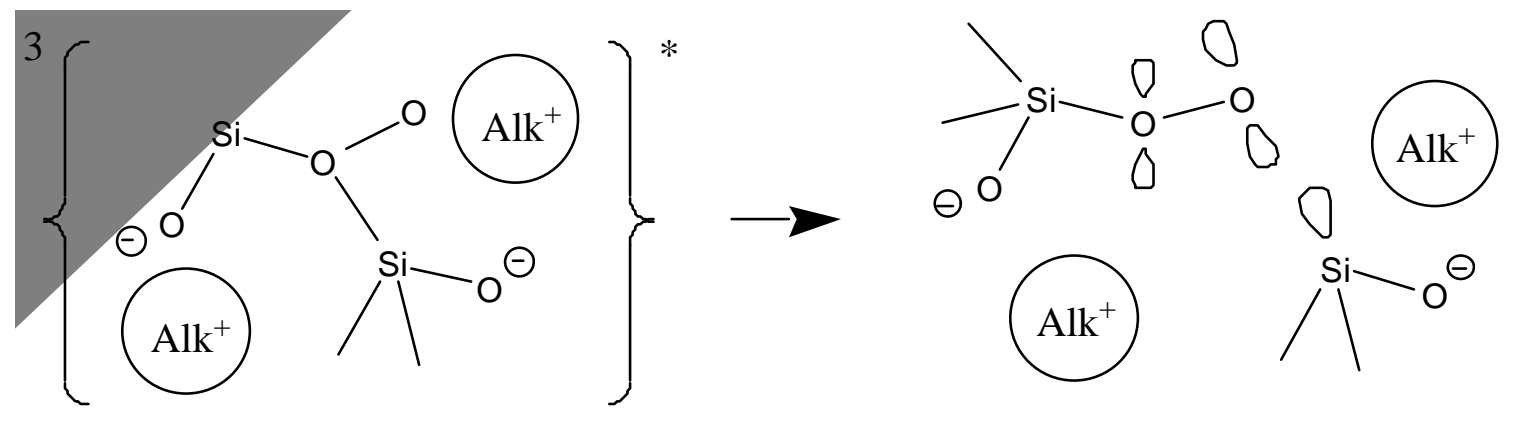

Interstitial superoxide anion, a precursor of oxygen molecule, is formed when this Sibonded peroxy radical reacts with a nearby $\equiv \mathrm{SiO}^{-} \mathrm{Alk}^{+}$group and the silica network rebuilds itself:

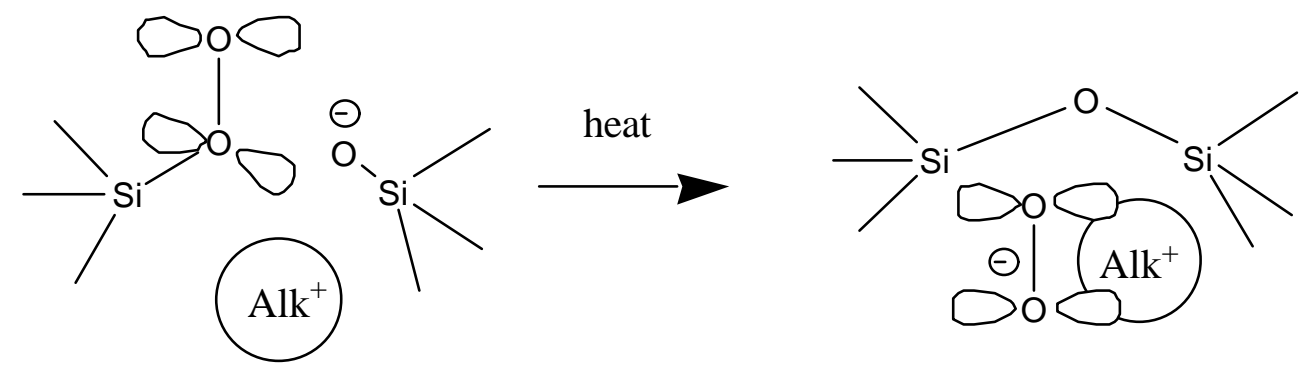

This thermally activated reaction is vigorous at $200 \div 300^{\circ} \mathrm{C}$, the expected temperature in the HLW repository. In silica (which does not yield oxygen bubbles), silicon peroxy radicals do not dissociate due to the lack of the $\equiv \mathrm{SiO}^{-} \mathrm{Alk}^{+}$groups; the stability of peroxy radicals decreases with the glass basicity. After recombination with a hole, the superoxide anion yields an $\mathrm{O}_{2}$ molecule. This reaction sequence accounts for the slow generation of interstitial $\mathrm{O}_{2}$ and evolution of oxygen bubbles in radiolysis of alkali oxide glasses. 


\subsection{Alkali Borates.}

Irradiation of borate and borosilicate glasses yields several classes of stable paramagnetic centers: (i) several variants of oxygen hole centers, (ii) electron centers (boron dangling bonds, hydrogen atoms, and alkali metal clusters), and (iii) boron and oxygen deficiency centers (such as peroxy radicals).

All of the observed hole centers have the alkali cation vacancy as a precursor $\left(\mathrm{O}_{1}{ }^{-}\right.$ defect). Some of the hole-trapping species are $>\mathrm{BO}^{-}$groups and distorted $\mathrm{B}_{4}-\mathrm{O}_{-}-\mathrm{B}_{3}$ linkages, other are $-\mathrm{BO}_{3}{ }^{2-}$ groups.

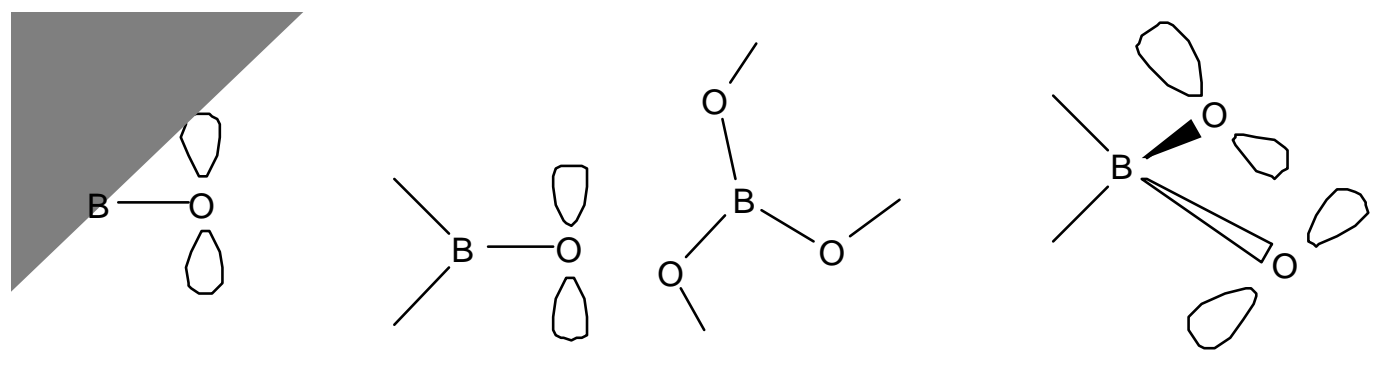

The $\mathrm{B}_{4}-\mathrm{O}-\mathrm{B}_{3}$ linkage at the cation vacancy is the main precursor of the hole center at low alkali loading; at higher loading, most of these hole centers originate from dissociated $>\mathrm{BO}^{-} \mathrm{Alk}^{+}$ groups. After hole trapping, tetrahedrally coordinated boron in the $\mathrm{B}_{4}-\mathrm{O}-\mathrm{B}_{3}$ linkage retracts and becomes trigonal. At very high alkali metal loading, the holes are trapped by $>\mathrm{BO}_{2}{ }^{2-}$ groups to yield $>\mathrm{B}\left(\mathrm{O}^{\bullet}\right) \mathrm{O}^{-} \mathrm{Alk}{ }^{+}$centers. Thermal activation of these hole centers at $100 \div 200^{\circ} \mathrm{C}$ results in the release of alkali cation and the formation of $>\mathrm{BO}_{2}{ }^{-}$centers in which the charge and spin are divided between two non-bridging oxygens. Density functional calculations indicate that stabilization of this center is due to strong electron correlation.

A typical example of the hole center in borate/borosilicate gass is given by a $\equiv \mathrm{BO} \bullet$ radical in mineral danburite (see below). The hole is trapped by a $\mathrm{Ca}^{2+}$ vacancy at the $\mathrm{B}_{4}-\mathrm{O}-\mathrm{B}_{4}$ linkage. One of the B-O bonds elongated to $0.245 \mathrm{~nm}$, and the boron becomes trigonal with quadrupole coupling constant $\mathrm{Q}_{\mathrm{cc}}$ of $2.77 \mathrm{MHz}$ and asymmetry factor of 0.11 . Quadrupolar and hyperfine dipolar tensors are coaxial within $10^{\circ}$ : 

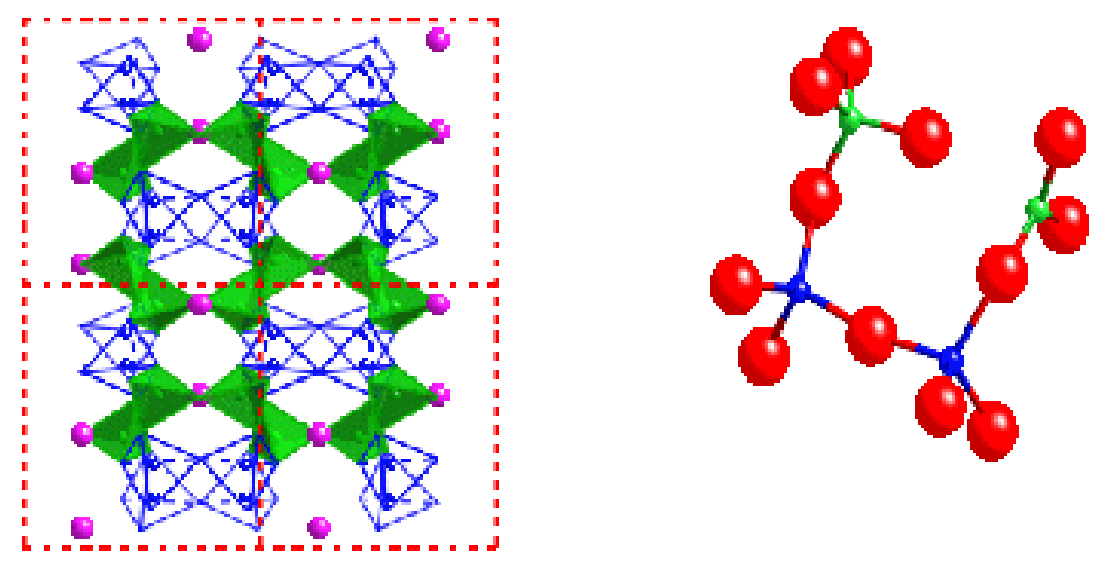

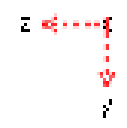

While the holes are trapped by several variants of the $\mathrm{O}_{1}{ }^{-}$defect, the electrons are trapped by overcoordinated oxygen $\left(\mathrm{O}_{3}{ }^{+}\right)$defects. If this defect involves a proton, an $\mathrm{H}$ atom is formed. If this defect involves a network boron atom, boron dangling bond center is formed.

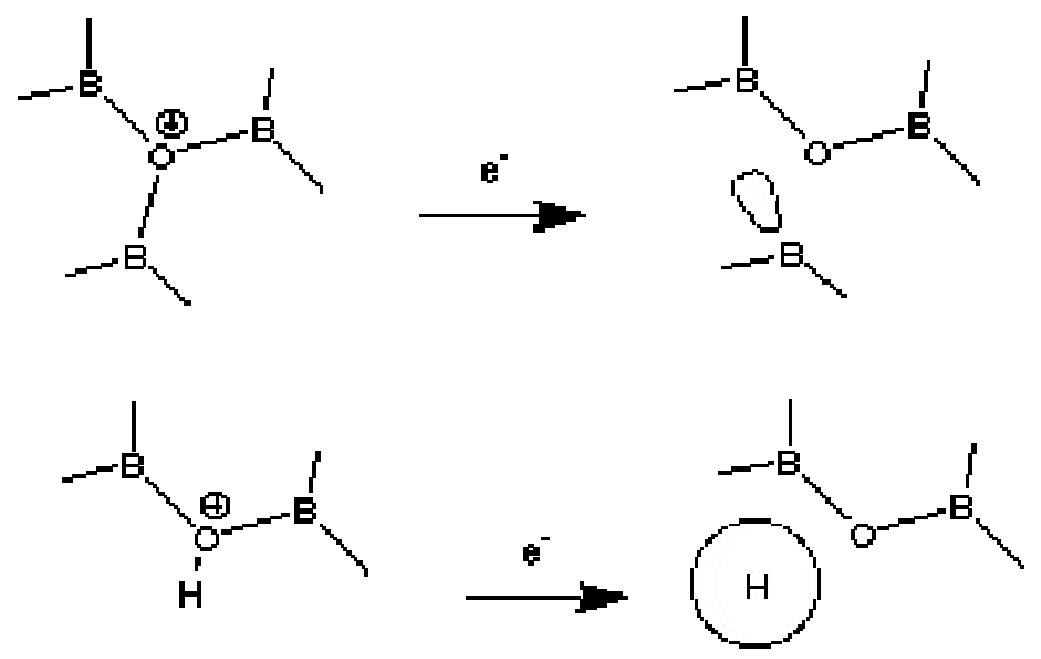

In alkali-loaded glasses, clusters of alkali cations bound to several $\mathrm{BO}_{4}{ }^{-}$groups may trap one or two electrons, yielding paramagnetic or diamagnetic centers, respectively. The formation of these centers requires thermal activation: the $\mathrm{O}_{3}{ }^{+}$defects are deeper electron traps. Long irradiation exposure also yields boron and oxygen deficiency centers, respectively: 

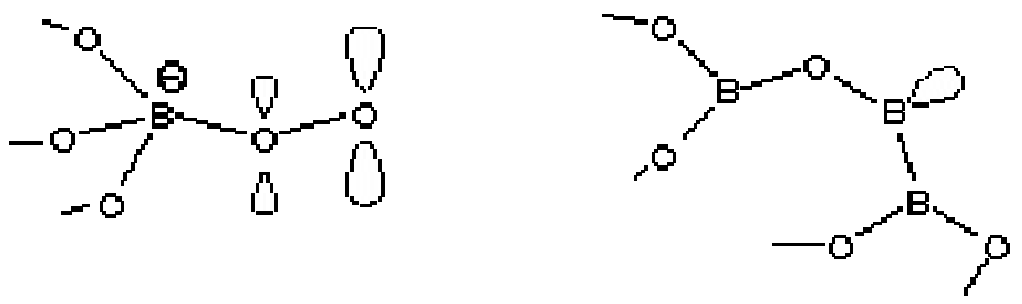

This boron peroxy radical is likely to be the precursor of interstitial oxygen in irradiated alkali borate glasses.

Most of radiation-induced defects in alkali silicates and borates were also observed in more complex borosilicate glasses. Our studies of the defect geometry and electronic structure yield the atomic-scale picture of the radiation damage in the HLW glass forms.

\subsection{Alkali Borosilicates.}

Due to limited miscibility of silicate and borate phases in the melt, most of alkali borosilicate glasses are microscopically phase separated. A common example is "pyrex" in which $1-5 \mathrm{~nm}$ borate islands are separated by pure $\mathrm{SiO}_{2}$. This microstructure accounts for the exceptional chemical durability of Pyrex glass.
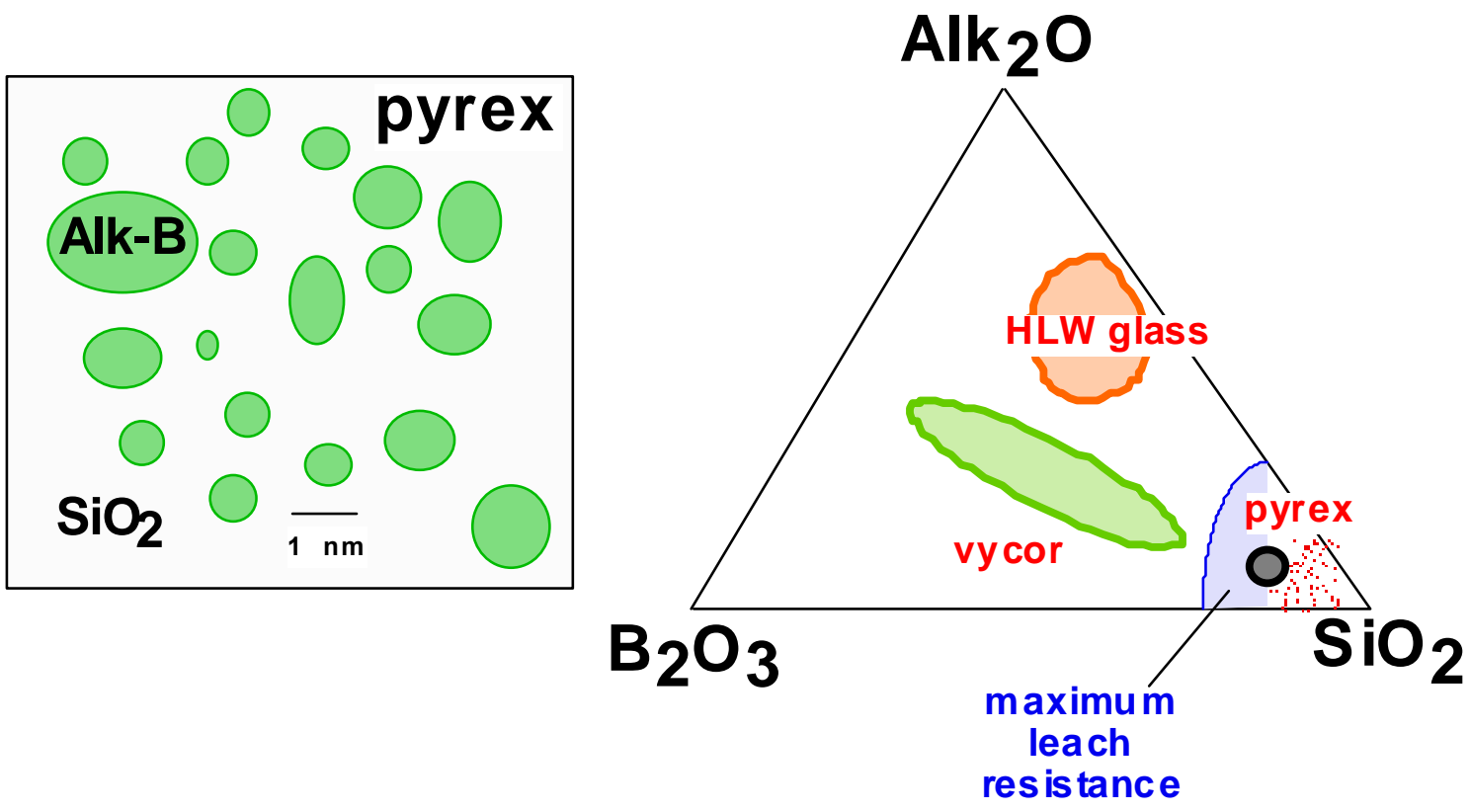

We demonstrated that in irradiated pyrex and vycor glasses, electron and hole centers cluster on the surface of borate phase islands, whereas oxygen deficiency/excess centers occur mainly in the silicate phase and have remarkably low yields. The only defect that occurs in both 
of the phases is hydrogen atom. Since the silicate network is largely spared the radiation damage, it retains the superior mechanical and chemical properties of undamaged glass.

Our results indicate that microscopically phase-separated glasses exhibit not only the highest leach resistance but also the highest radiation resistance among borosilicate glasses. Such phase separation is beneficial for the HLW glass and should be sought after.

\section{Short-lived reactive intermediates: Hydrogen atoms.}

Due to their small size, hydrogen $(\mathrm{H})$ atoms are the only neutral species that rapidly migrate in glasses. These $\mathrm{H}$ atoms are also very reactive species that account for numerous annealing reactions with radiation-induced defects. Using time-resolved pulsed electron paramagnetic resonance, the $\mathrm{H}$ atoms were observed from $10 \mathrm{~ns}$ to $1 \mathrm{~ms}$. We use spinpolarized $\mathrm{H}$ atoms to probe the glass structure and to study other short-lived intermediates. Previously, we have studied the H atoms in pure silica glass (I. A. Shkrob and A. D. Trifunac, Phys. Rev. B 54 (1996); J. Chem. Phys. 107 (1997) 2374); lately we expanded this study to vitreous $\mathrm{B}_{2} \mathrm{O}_{3}$. We found that

- The H/D atoms are spin-polarized due to $\mathrm{H}+\mathrm{H}$ recombination in radiolytic spurs. $\mathrm{H}_{2}$ formed in radiolysis is also from this reaction.

- Spin relaxation in these H/D atoms is due rapid hopping between the sites with different arrangements of magnetic boron nuclei. From the temperature dependence of the relaxation times, we determined the activation energy of hopping $(0.13 \div 0.16 \mathrm{eV})$ and the residence time of the $\mathrm{H}$ atom in a trapping site $(5 \div 7 \mathrm{~ns}$ at $300 \mathrm{~K})$; the diffusion coefficient is $1.5 \times 10^{-7}$ $\mathrm{cm}^{2} / \mathrm{s}$ at $300 \mathrm{~K}$ and the mean hoping distance is $0.56 \mathrm{~nm}$.

- Free $\mathrm{H}$ atoms that escape from the spurs slowly recombine with each other and with metastable radiation-induced centers. Below $140 \mathrm{~K}$, trapped $\mathrm{H}$ atoms reside in a cavity formed by two or three B-O-B bonds; in high- $\mathrm{OH}$ loaded glass there are also $\mathrm{H}$ atoms trapped at a site formed by two $\mathrm{HO}$-terminated boroxol rings.

- There is no isotope effect on the diffusion of H/D atoms in boron and silicon oxide glasses; there is a considerable isotope effect on their yield above $20^{\circ} \mathrm{C}$. Our results and calculations indicate that the isotope effect on the H/D atom yield is due to electron trapping by pre-existing $>\mathrm{OH}^{+}$defects. 


\section{Conclusions and practical implications.}

Several families of radiation-induced defects in oxide glasses have been characterized structurally on the atomic scale. Chemical pathways leading to these defects are established. All in all, our study points out to remarkable robustness of mixed oxide glasses as waste storage forms. It appears that concerns about radiation stability of these glasses (in particular, the occurrence of radiation-induced phase separation and mass migration of cations) are exaggerated. We emphasize that only the effects of $\beta$ and $\gamma$ radiation were examined in our study. These two types of radiation are expected to dominate in the first Kyr of storage. By the "radiation stability" we imply the effect of the ionizing radiation on the HLW glass prior to contact with seeping water.

We found no evidence for enhanced alkali cation migration in irradiated glasses (invoked as possible mechanism for extensive radiation damage). In alkali silicates, trapping of holes by nonbridging oxygens does not release these cations. In alkali borates, the holes are trapped by pre-existing cation vacancies. There is no evidence for efficient cation emission due to hole trapping or extensive metallization due to electron trapping. While this metallization is known to happen in ionic crystals, in boron-containing glasses, due to their more covalent nature, the electrons are preferentially trapped by over-coordinated oxygen, yielding dangling bond centers. Thus, concerns about explosive relaxation of defects in the metallized glass are exaggerated.

In microscopically phase-separated borosilicate glasses the radiation damage is concentrated at the boundaries between the silicate and borate domains. The damage to the silica matrix is minimal. The microscopic separation increases the radiation durability in the same way it increases chemical durability. Since most borosilicate HLW glasses are phase separated, the matrix itself inhibits the growth of radiation-induced defects.

Transport properties of mobile $\mathrm{H}$ atoms in room-temperature silicon and boron oxide glasses have been studied. These atoms are shown to diffuse in glass nearly as rapidly as in water and efficiently anneal radiation-induced points defects. Our study suggests that hydroxyl groups $(<2000 \mathrm{ppm})$ are beneficial for the radiation stability of HLW forms. Possibly, some metals might be added to the glass frit as hydroxides (this would also reduce the melting point of glass).

Formation of molecular oxygen in glasses subjected to high-dose irradiation has been studied. It is shown that there is no efficient formation of $\mathrm{O}_{2}$ under high-energy irradiation ( $>1$ $\mathrm{MeV}$ ). Our study reaffirms low-end estimates of volatilization in the HLW glasses (10 Kyr of storage as the onset of the bubble formation).

\section{Recommendations for further studies.}

Short of the catastrophic failure of a geologic repository, the storage of HLW in the first 1-10 Kyr will be safe. However, prognostication of the HLW glass behavior after the first 10 Kyr of storage, under various "catastrophic" scenarios (collapse of this repository, flooding, 
etc.), requires experimental approaches that do not presently exist. While our study supports the view that borosilicate glass is an adequate choice of the HLW matrix for storage in the nearest future $(<1 \mathrm{Kyr})$, the robustness of this matrix for storage over $>10 \mathrm{Kyr}$ is questionable. In 10$100 \mathrm{Kyr}$, extensive radiation damage will compromise the glass integrity. No mitigation of this damage is possible.

In this respect, $\mathfrak{t}$ is important to follow up this work, as there are no experimental studies on the very long term radiation and chemical stability of glasses. In particular, little is known about the effect of prolonged $\alpha$ radiolysis, while this type of radiation will prevail after the first Kyr. Furthermore, effects of radiation damage on the leach resistance of glasses need to be assessed. As only microscopic samples can be irradiated to Tera-rads over short period of time, using focussed particle beams, the development of suitable analytical and spectroscopic techniques should be requested. 